\title{
COMMENTARY
}

\section{Cigarette Smoking among High School Seniors: Did the Rate Decline?}

\author{
PATRICK M. O'MALley
}

Institute for Social Research, University of Michigan, Ann Arbor, Michigan 48106

\begin{abstract}
Some questions about adolescent smoking levels as reported in national surveys were recently raised by M. B. Mittelmark, D. M. Murray, R. N. Luepker, and T. F. Pechacek, [Prev. Med. 11, 708-712 (1982)]: such levels may be underreported, calling into question the decline in adolescent smoking. The present article suggests that the most plausible interpretation of available data is that smoking is, in general, validly reported and that there was a real decline in smoking rates among high school seniors during the period 1977-1981. (C) 1984 Academic Press. Inc.
\end{abstract}

\section{INTRODUCTION}

Mittelmark and colleagues (8) have presented what they believed to be "new data which point to sharply higher smoking levels than those reported elsewhere"' (p. 708). They suggest that two national surveys $(2,5)$ had likely underreported cigarette smoking rates by adolescents; they further suggested that reported declines in smoking rates in recent years may have reflected an increase in underreporting, rather than a decline in actual use. Two kinds of evidence are mentioned by Mittelmark et al. in support of their thesis of underreporting in the national surveys: One is that the national surveys report lower rates than found in surveys of Minnesota students; the second is based on other research which has shown that local surveys using validation procedures obtain higher estimates of smoking than do comparable surveys using only self-report procedures.

The purpose of this note is to present some evidence indicating that adolescents do give valid reports of their smoking behavior, at least under some conditions, and that the recent declines in reported use most likely reflect actual smoking behavior. We will approach this goal in three ways: (a) by citing data from one major ongoing national survey (6) which do not show very different smoking rates than the data reported by Mittelmark et al. (8); (b) by demonstrating that the evidence that validation procedures generally produce significantly higher reported smoking rates is questionable; and (c) by showing that some recent national trends in variables other than self-reported smoking do not support a hypothesis of an increase in underreporting.

\section{ARE NATIONAL SURVEY RATES LOWER?}

We can add little to the published report by Green (5), but we would like to comment on whether the data presented by Mittelmark et al. do, in fact, show "sharply higher smoking levels" than those reported from the Monitoring the Future project (which provided the data for the Bachman et al. (2) report). First, a brief description of the two studies is in order.

The Minnesota data presented by Mittelmark et al. came from a September 
1980 survey of all 7th through 11th grade students in three schools in the Minneapolis-St. Paul area. The students first viewed a videotape which described how smoking levels could be measured by analysis of saliva or expired air, provided samples of saliva and air, and then filled out a confidential questionnaire on their smoking behavior.

The Monitoring the Future project conducts annual surveys of licit and illicit drug use among national samples of high school seniors. Since 1975 , from 17,000 to 19,000 seniors in approximately 125 schools per year have participated. The data on drug use and related attitudes and behaviors are obtained via self-completed confidential (not anonymous) questionnaires filled out in classroom settings, administered by representatives of the Institute for Social Research. [For more details on survey procedures, see Ref. (6).]

Because of the differences between the two studies, it is difficult to make direct comparisons of their findings. Both the questions and the samples of the two studies are quite different. The Monitoring the Future sample is intended to be representative of 12 th graders nationally, while the Minnesota data are intended to be representative of 7 th-11th graders in a single school district. To make the samples more comparable, we will restrict our attention to only the eldest students (15-16 years old) in the Minnesota data. We could restrict the Monitoring the Future sample to white respondents living in non-self-representing SMSAs in the North Central region, which would more closely approximate the Minnesota sample's demographics, but smoking rates in that restricted sample are very close to rates for the sample as a whole. (As Bachman et al. (2) reported, of the four geographical regions in the United States, the North Central region showed the smallest departures from the average for the nation as a whole in use of various substances, including cigarettes. More specifically, for 30-day prevalence of cigarette smoking, the Northeast, North Central, and South are all about equal, with the West distinctly lower.)

Mittelmark et al. report a current prevalence of cigarette smoking-defined as "smoked at least a few times per month"- of 33.2\% among 15- to 16-year-old students surveyed in September 1980. The Monitoring the Future data for seniors (most of whom were 17 or 18 years old) surveyed in the spring of 1980 and 1981 show current prevalence rates-defined as smoking at least one cigarette in the previous 30 days - of 30.5 and $29.4 \%$, respectively. An additional $7.3 \%$ (average for 1980 and 1981) did not smoke at all in the previous 30 days, but identified themselves as "occasional, but not regular" smokers. Since some of these would likely have said they smoked at least a few times a month, the Monitoring the Future figures seem quite comparable to the Minnesota figure.

One might argue that the Monitoring the Future data should show higher rates since the respondents are older, but it is not clear that there would be substantially more smokers at the rather light smoking level under consideration. Furthermore, the Minnesota sample includes some respondents who will later drop out of school, and cigarette smoking is distinctly higher than average among this group (3).

In sum, cigarette rates do not appear to be significantly lower in the Monitoring the Future data than in the Minnesota data. 


\section{EVIDENCE ON VALIDATION PROCEDURES}

With respect to data on the validity of self-reports of cigarette smoking by adolescents, Mittelmark et al. cite two studies as showing that "self-report data significantly underestimated cigarette use" (p. 709). But one of those studies (7) did not in fact find statistically significant differences in self-reported smoking rates between two groups, one of which used the "bogus pipeline" methodology. ${ }^{1}$ The difference was in the expected direction (higher smoking rates in the bogus pipeline group), but was not statistically significant. The other study cited involved a total of 170 children with an average age under 14 years and did find signficantly more smoking in bogus pipeline groups compared to questionnaireonly groups (4).

Since the Mittelmark et al. report, another study that examines the issue of the validity of self-reports of smoking among adolescents has appeared. Akers $e t$ al. (1) surveyed over 2,000 students in grades 7 through 12 in one community in each of 2 years. All respondents in both years completed a confidential questionnaire and a randomized response instrument. In addition, saliva specimens were collected from sub-samples of the students. Some of these $(n=577)$ were ;subjected to biochemical analysis for thiocyanate in year 1; in year 2, 850 saliva specimens were drawn but not analyzed. Prior to the collection of the saliva, the respondents were told that the purpose was to check the accuracy of their responses in the questionnaires. The questionnaires, the randomized response instrument, and the saliva specimens were administered in four different sequences in year 1 and in two different sequences in year 2 . Thus, rates of cigarette use reported in the questionnaire could be compared to rates obtained via the randomized response instrument and the biochemical measure of thiocyanate. Rates could also be compared between the questionnaire with and without the bogus pipeline.

The findings from this well-designed study were quite clear: "The pipeline conditions seem neither to counter under-reporting nor to induce over-reporting" [Ref. (1), p. 246]. Akers et al. concluded that "adolescents in this study reported their smoking behavior quite accurately" (p. 246).

One other piece of information is relevant. If there were substantial underreporting of cigarette use by seniors, it seems probable that there would be considerably less tendency to underreport among older respondents. One component of the Monitoring the Future project is a longitudinal follow-up of random samples of approximately 2,400 participants from each senior class. Mail-out questionnaires are sent to members of each panel for several years after high school and are returned by approximately $80 \%$ of each panel. Data from the panels from the classes of 1976 to 1981, follow-up in 1977 through 1982, show no significant increase in monthly prevalence of cigarette use through age 24; other measures of cigarette smoking show increased amounts of smoking, but the proportion reporting any smoking in the $\mathbf{3 0}$ days prior to the survey is essentially unchanged

\footnotetext{
1 "Bogus pipeline" refers to a situation where subjects are made to believe that their self-reports of behaviors or attitudes can and will be verified by the researcher; the term "bogus" is used because the researcher does not actually verify the self-reports.
} 
(9). The lack of increase is contrary to what would be expected if seniors were underreporting.

In sum, there appears little doubt that adolescents report their smoking behavior accurately, at least under some conditions. Under other conditions reports may not be so accurate. If, for example, respondents are not convinced that their reports will be kept confidential, they might be tempted to underreport, and a bogus pipeline might help counter that temptation. Or, perhaps younger students are more likely to underreport. This might explain why Evans $e t$ al. found a difference in their study of 13- to 14-year-old students (4).

This hypothesis could be tested: the amount of bias in self-reports compared with validated reports should be greater for younger than for older students. There is some support for this notion. The discrepancy between data reported by Green (5) and data reported by Mittelmark et al. (8), as measured by the ratio of reported rates, is greater for those 12-14 years old than for those 15-16 years old. Pechacek et al. (10) report an age-linked increase in the association between selfreports and biochemical measures of smoking. On the other hand, Akers et al. (1) found no evidence that there was any bias in either their junior or their senior high school sample. They attribute the lack of bias to the fact that their respondents were satisfied that their responses would remain confidential, and thus, they tended to answer truthfully. Overall, the data reviewed here suggest that there is probably very little underreporting by older students, with perhaps some underreporting in questionnaires by younger students who are not convinced that their responses will be kept confidential.

\section{COMMENTS ON TREND DATA}

The other major question raised by Mittelmark et al. (8) is one of trends. Bachman et al. (2) and Johnston et al. (6) have reported a decline in cigarette smoking among seniors between 1977 and 1981. Mittelmark et al. suggest that, rather than a decrease in use, there has been an increase in the tendency to underreport. Following Warner (11), they suggest that increased awareness of the health risks of smoking and the decreased social acceptability of smoking may have led to more underreporting. This explanation seems unlikely for several reasons.

Perhaps the most compelling reason is that reports by seniors of the proportions of their friends who smoke show trends very parallel to their reports of their own use (Fig. 1). If concealing were a significant factor in the decrease in self-reported use, there would presumably be much less tendency to distort reported use by others. Over time, there would be an increased gap between one's own reported use and use by "most or all" of one's friends (with friends' use relative to own use becoming greater). In fact, the trends in these two measures are very similar; to the extent that they do diverge, it is in a direction opposite to that expected under an increased concealment hypothesis. The percentage who say that none of their friends are smokers has increased (data not shown), as would be expected if fewer seniors were smoking. It is noteworthy that both self-reported and friends' use increased between 1981 and 1982; there is less reason to believe that any underreporting tendency would have altered its trend than that there was actually an increase in smoking rates. 


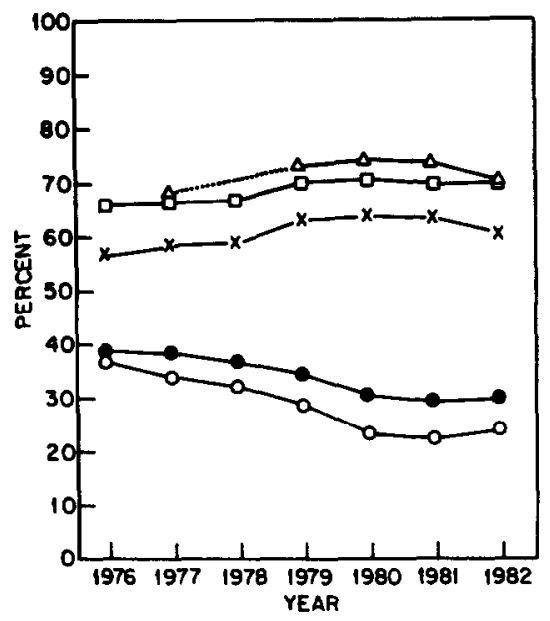

FIG. 1. Trends in prevalence of cigarette smoking and related measures. The dotted lines connect points whose values have been adjusted to correct for context effects. See Ref. (6) for complete information on these measures. Key: percentage of seniors who say $\Delta$, their friends would disapprove of their smoking; $\square$, they disapprove of smoking; $\times$, smoking entails a great risk of harm; 0 , they used cigarettes at least once in the prior 30 days; $O$, most or all of their friends smoke.

Based on the explanation advanced by Mittelmark et al. (8), there should be an increase in perceived health risks associated with smoking concomitant with decreases in self-reported smoking rates. Of course, any decrease in self-reported smoking accompanied by an increase in perceived health risks could also very easily be reflecting reality; however, the explanation advanced by Mittelmark $e t$ al. more strongly implies contemporaneous divergence. There was in fact an increase in perceived health risks between 1976 and 1979, followed by a stability until 1982, when it decreased. Monthly prevalence of cigarette use did not entirely parallel this trend; it decreased slightly between 1976 and 1978 (by $2.1 \%$ ), somewhat more by 1980 (down $6.2 \%$ from 1978), and remained at the same level in 1982. Figure 1 shows graphically that the trend lines do diverge between 1976 and 1979, but that reported use continued to decline through 1981 while perceived risk of harm did not increase further.

Relevant to the decreased social acceptability, there was very little change between 1979 and 1981 in the disapproval of smoking, either by seniors themselves ( 70.3 vs $69.9 \%$ ) or by their friends ( 73.4 vs $73.8 \%$ ); nevertheless, selfreported use dropped substantially during that interval (34.4 to $29.4 \%$ ).

In addition to the above data which seem inconsistent with the suggested explanation of Mittelmark et al., it seems intuitively unlikely that the recent trends in cigarette smoking could be entirely due to deliberate underreporting, given the quite varied trends seen in use of other substances; e.g., marijuana use increased through about 1979, and has since decreased, and alcohol use has been stable at very high levels of use. Both of these behaviors are reported by higher proportions of seniors for lifetime and annual measures, compared to cigarettes (6). Certainly, marijuana and alcohol have received their share of bad publicity in the past few years. The disparity in trends for these and various other substances makes it 
unlikely that seniors would single out cigarette use to underreport. The high levels of reported use for cigarettes and other, more disapproved behaviors make the recent trends in cigarette use seem more likely to be factual than artifactual.

Although the rate of cigarette use among seniors probably did decline between 1977 and 1981, the more recent data (through 1983) unfortunately show no continuance of that decline. Thus, as Mittelmark et al. quite correctly observed, it would indeed be a serious public health error to conclude that the adolescent smoking problem is now lessening.

\section{REFERENCES}

1. Akers, R. L., Massey, J., Clarke, W., and Lauer, R. M. Are self-reports of adolescent deviance valid? Biochemical measures, randomized response, and the bogus pipeline in smoking behavior. Soc. Forces 62, 234-251 (1983).

2. Bachman, J. G., Johnston, L. D., and O'Malley, P. M. Smoking, drinking, and drug use among American high school students: Correlates and trends, 1975-1979. Amer. J. Public Health 71, 59-69 (1981).

3. Bachman, J. G., O'Malley, P. M., and Johnston, J. "Youth in Transition," Vul. 6. Institute for Social Research, Ann Arbor, Mich., 1978.

4. Evans, R. I., Hansen, W. B., and Mittelmark, M. B. Increasing the validity of self-reports of smoking behavior in children. J. Appl. Psychol. 62, $521-523$ (1977).

5. Green, D. E. "Teenage Smoking: Immediate and Long-Term Patterns." National Institute on Education, Washington, D.C., 1979.

6. Johnston, L. D., Bachman, J. G., and O'Malley, P. M. "Student Drug Use, Attitudes, and Beliefs: National Trends 1975-1982." National Institute on Drug Abuse, Washington, D.C., 1982.

7. Luepker, R. V., Pechacek, T. F., Murray, D. M., Johnson, C. A., Hund, F., and Jacobs, D. R. Saliva thiocyanate: A chemical indicator of cigarette smoking in adolescents. Amer. J. Public Health 71, 1320-1324 (1981).

8. Mittelmark, M. B., Murray, D. M., Luepker, R. V., and Pechacek, T. F. Cigarette smoking among adolescents: Is the rate declining? Prev. Med. 11, 708-712 (1982).

9. O'Malley, P. M., Bachman, J. G., and Johnston, L. D. "Period, Age, and Cohort Effects on Substance Use among American Youth: 1976-1982," Monitoring the Future Occasional Paper No. 14. Institute for Social Research, Ann Arbor, Mich., 1983.

10. Pechacek, T. F., Murray, D. M., Luepker, R. V., Mittelmark, M. B., Johnson, C. A., and Shultz, J. M. Measurement of smoking behavior: Rationale and methods. J. Behav. Med. 7, 123-140 (1984).

11. Warner, K. E. Possible increases in the underreporting of cigarette smoking. J. Amer. Stat. Assoc. 73, 314-318 (1978). 